\title{
New Roles for Psychologists in Medical Settings: Direct Services in a Hong Kong Accident and Emergency Department
}

\author{
Wo Oi Kwok ${ }^{1}$, Christopher D. Tori ${ }^{1}$, Timothy H. Rainer ${ }^{2}$ \\ ${ }^{1}$ California School of Professional Psychology, Alliant International University, Hong Kong, China \\ ${ }^{2}$ Accident and Emergency Medicine Academic Unit, Chinese University of Hong Kong, Hong Kong, China \\ Email: rkwok@cuhk.edu.hk
}

Received February 3, 2013; revised March 7, 2013; accepted March 15, 2013

Copyright (C) 2013 Wo Oi Kwok et al. This is an open access article distributed under the Creative Commons Attribution License, which permits unrestricted use, distribution, and reproduction in any medium, provided the original work is properly cited.

\begin{abstract}
Study Aim: Descriptions of the direct services of a psychologist (i.e. clinical psychology intern) in an accident and emergency department (AED) are provided as a new and rare area of professional practice. Method: Data were collected in a large, Hong Kong, government hospital over an eighteen-month period. Recipients included 281 patients, 150 medical staff members, and 44 relatives of patients. Results: Interventions with patients and their family members centered on crisis management, diagnostic assessment, psychoeducation, and consultations regarding long-term adjustment to medical infirmities. The majority of staff members sought consultations regarding work related stress such as burnout symptoms and coping with vicarious trauma experiences. Frequency tables provide information on utilization of specific services while case vignettes contextualize psychological interventions. Conclusion: There are many potential benefits of psychological service for AED patients and staff.
\end{abstract}

Keywords: Accident and Emergency Departments; Psychological Services Utility; Patients and Staff

\section{Introduction}

From its academic and experimental origins, psychology rapidly developed clinical applications in a wide variety of settings with the most recent being medical departments in hospitals and clinics [1,2]. While psychotherapeutic, assessment, and consulting services of clinical psychologists in health centers were initially psychiatric in nature [3], as the profession matured, psychological services began to be offered in other medical departments such as neurology [4], pediatrics [5], cardiology [6] and oncology [7] to name just a few.

Only recently, however, psychologists have been employed in accident and emergency departments (AEDs) with only a single book chapter describing the types of services rendered in this challenging milieu [8]. Given this hiatus in the literature, the work of a psychologist in the AED of a large teaching hospital located in Hong Kong, China was recorded. It is hoped that information provided may be useful for hospital administrators and practitioners regarding new avenues to improve psychosocial services for patients and staff members in AED or similar medical departments worldwide.
In his historical review of emergency medicine, Bodiwala [9] noted that this specialty involves the provision of lifesaving interventions to critically ill persons using multidisciplinary medical approaches. According to Suter [10], during 1950s and early 1960s the need for medical specialists dealing with emergencies was recognized. In England, groups of "casualty surgeons" were established and as early as 1952 which, in turn, led to the establishment of the Casualty Surgeons Association in 1967. This group later became the British Accident and Emergency Association. Similar professional developments regarding emergency room services also began occurring in the United States and then spread internationally.

There is general agreement that AED professional services require knowledge and proficiencies related to the prevention, diagnosis, and management of acute and vital aspects of illness or injury [11]. Specifically, services involve, "the full spectrum of episodic undifferentiated physical and behavioural disorders; they further encompass an understanding of the development of prehospital and inhospital emergency medical systems and the skills necessary for this development” [12]. 
In 1947, the first Hong Kong casualty unit was established at Queen Mary Hospital which became an AED in 1983 [13]. There are currently 16 AEDs in Hong Kong operating on a daily and continuous basis. According to the Hospital Authority Statistical Report, there were 2.2 million visits to AEDs between 2010 and 2011 [14].

A five category triage system is used to prioritize treatment for all incoming patients to AEDs: 1) critical lifesaving interventions are needed; 2) immediate emergency services are required; 3) urgent but not immediate care is necessary; 4) semi-urgent treatment is indicated; and 5) non-urgent care is necessary. According to statistics of the hospital authority in 2009/2010, 32\% emergency patients were classified as either critical or urgent with the remaining $68 \%$ being in the other triage categories [15].

Because emergency medicine is an internationally practiced specialty, it is essential that interventions be culturally sensitive to local demands and regional manifestations of disease. For example, most Chinese people place high value on harmonious relationships and, therefore, tend to be deferent to authority figures. Not surprisingly, Zhang et al. [16] found that only 11\% of Chinese patients played an active role in making treatment decision. Also, in Asian populations, somatization of psychosocial problems is common [17]. Thus, having someone with special expertise regarding the social-cultural manifestations of serious illness or injury may be very helpful for accident and emergency department medical personnel.

In addition to crisis management and disaster related triage services, roles of clinical psychologists in urgent medical situations are expanding [18]. Direct services to patients often include psychological assessments (e.g., mental status examinations, suicidal risk assessment, danger to others evaluations), assistance in adjusting to critical medical conditions (e.g., psychoeducation regarding illness management), individual and family counseling regarding adjustment to medical conditions and life style changes, case consultation with primary care physicians regarding cultural and psychological aspects of various symptoms, and liaison with other medical specialties (e.g., psychiatry, social welfare, physical therapy).

Given the empirical literature concerning secondary traumatic stress and vicarious traumatization among health workers [19,20], it would be surprising if these sort of problems were lacking among AED professional staff. In like manner, burnout symptoms (e.g., fatigue, dejection, and irritability) would likely be found among an overburdened professional staff [21]. In order to promote a healthy working environment, psychological services for staff could include stress reduction programs, brief emotional support, career change consultations, and individual debriefing counseling following mortality experiences.
This research describes the direct services provided by a psychologist in an AED as a guide to those who might want to provide direct psychosocial interventions in similar settings. In order to accomplish this end, the services of a psychologist working in an AED were quantified and case vignettes were recorded. Finally, it is hoped that findings from the present study will illustrate the interaction of medical and psychological domains and reveal specific ways social science and medical practitioners can collaborate in the care of patients experiencing acute biopsychosocial distress.

\section{Method}

\subsection{Study Design and Setting}

This illustrative and retrospective study was implemented from 1 July 2009 to 28 February 2011 in the Emergency Department of the Prince of Wales Hospital (PWH) in collaboration with the Accident and Emergency Medicine Academic Unit of the Chinese University of Hong Kong. Ethical approval was obtained from relevant institutional review boards as this investigative protocol complied with the stipulations of the Declaration of Helsinki.

\subsection{Participants}

Direct services were provided by a psychologist to three groups 1) patients, 2) relatives of patients, and 3) professional staff members.

There were 281patients receiving 284.4 hours of care (average session length approximately one hour). In this sample, 217 (77.2\%) were women and 64 (22.8\%) were men with age ranging from 13 to 96 years $(M=55.99$, $S D=22.30$ ) and virtually all being ethnic Chinese (99.1\%). Reasons for admissions included traumatic (head) injuries, medical conditions requiring immediate care, obstetric emergencies, chronic health problems, confirming disease diagnoses, suicide attempts, acute and chronic psychological distress or mental deterioration.

Forty-four relatives of patients each received one hour of care. Demographic information on this sample was not archived as these individuals were not hospital patients and medical records were not initiated. At the same time, however, these individuals often had very important care giving duties for the identified patient.

Direct services were provided to 150 professional staff members (104 woman and 46 men) with the majority being health care professionals who provided direct patient services. Also included in this sample were some hospital managerial and administrative personnel. Reasons for consultations included career development issues, occupational stress, work conflicts, parenting pressures, relational problems, bereavement, and psychological assessment. As these individuals were not hospi- 
tal patients, various consent and confidentiality documents were not obtained to allow for a more free exchange during consulting sessions.

\subsection{Instruments}

\subsubsection{The Chinese Mini-Mental Status Examination (CMMSE)}

This instrument is a brief and practical clinical measurement including orientation, registration, recall, language, arithmetic ability in assessing cognitive functioning of elderly. It was originally designed by Folstein et al. [22] and was translated to various languages including Chinese [23]. Its internal consistency has been found to be excellent (Cronbach's $\alpha=0.86$ ) as was test-retest reliability, $r=0.78$.

\subsubsection{Montreal Cognitive Assessment (MoCA)}

This test was developed by Nasreddine et al. [24]. It has been used with Chinese samples with excellent psychometric proprieties being reported [25]. It is a brief clinical screening tool for the assessment of short term memory, visuospatial ability, executive functioning, attention, concentration, and orientation. Completing this instrument typically takes approximately 10 minutes.

\subsubsection{Chinese Sentence Completion Test}

This is a projective test used to explore emotions and cognitions that are often hidden from immediate recall. As with all other tests of this type, it is a free-response measure where respondents are asked to complete 50 sentence stems for the purpose of assessing temperament and self-development issues. For example, the first stem of the instruments is "I want to know..." which would be made into a complete sentence using any phrase that the respondent chooses.

\subsubsection{Bender Visual Motor Gestalt Test-II}

This instrument was developed by Lauretta Bender and is often used as a brief neurological screen assessing perceptual motor skills, motor development, and cognitive dexterity. Personality and emotional problems can be reflected on the test as well [26].

\subsubsection{Projective Drawings (Draw-a-Person, Kinetic-House-Tree-Person, Draw-a-Family)}

These instruments are projective tests where participants are asked to draw a person, objects, or a situation in order to assess the cognitive, psychological, emotional, and relational issues. They are widely used as brief screening tools in a variety of clinical settings [27].

\subsubsection{SAD PERSONS Scale}

This instrument was developed Patterson et al.[28] and is mainly used to predict suicidal risk using the variables of gender, age, mood, previous attempts substance abuse, rational thinking processes, social supports, suicidal plans, spouse specifics, and degree of illness and pain. It has been found to be very useful in emergency settings [29].

\subsubsection{Chinese Millon Clinical Multiaxial Inventory-III (MCMI-III)}

This test was initially developed by Theodore Millon as a bridge from population-based measures to idiographic utility. It is intended to reveal the relationship between personality and psychopathology in order to tailor appropriate treatment plans for individuals. The MCMI-III has been translated and validated for use with Chinese populations by Li et al. [30].

\subsection{Procedure}

Referrals to the psychologist were made by professional staff or by individual persons (i.e., "walk-ins”). Information regarding the availability of the psychologist was announced by the Chief of Service and the Department Manager to all staff. Following a brief introduction, the pager number of the psychologist was placed in the nurse station in the emergency department of PWH. Direct services were offered two days per week and the psychologist was provided office space in the AED. Physicians and nurses paged the psychologist directly when services were needed. Informed consent was obtained prior to service delivery. Patients and staff were not billed for consultations with the psychologist.

\section{Results}

\subsection{Quantitative Findings}

Table 1 provides a summary regarding the frequencies of initial direct psychotherapeutic services provided by the psychologist for patients and professional staff.

The most common service of the psychologist with patients concerned reassuring and informing people regarding their immediate medical problems (e.g., adjustment assistance and crisis management), stress reduction techniques, and helping patients develop daily living plans. Critical treatments included suicide prevention interventions and drug compliance and abuse counseling.

The services offered to the professional staff by the psychologist were as follows: career consultations $(n=5)$, reducing occupational stress $(n=126)$, and conflict or complaint resolution $(n=24)$. Staff member often spoke to the psychologist in a completely confidential manner about demanding workload issues, adequacy fears, occasional difficulties with patients and colleagues, vicarious traumatic experiences (e.g., grief reactions), and personal adjustment matters.

Table 2 gives the referrals made by the psychologist to 
Table 1. Frequencies of direct services psychotherapeutic services provided to individuals.

\begin{tabular}{|c|c|c|}
\hline Services & $\begin{array}{l}\text { Patients } \\
(N=281) \\
\text { frequency }\end{array}$ & $\begin{array}{c}\text { Staff } \\
(N=150) \\
\text { frequency }\end{array}$ \\
\hline Parental support & 5 & 7 \\
\hline ADL assessment & 59 & 0 \\
\hline Stress management & 55 & 3 \\
\hline Pain management & 20 & 0 \\
\hline Safety assessment & 8 & 0 \\
\hline Suicidal risk assessment & 46 & 1 \\
\hline Drug compliance assessment & 67 & 1 \\
\hline Anticipatory grief & 7 & 0 \\
\hline Brief psychological support & 185 & 8 \\
\hline Crisis management/psychology first aid & 30 & 3 \\
\hline \multicolumn{3}{|l|}{ Psychological adjustment } \\
\hline Newly diagnosis cancer & 4 & 0 \\
\hline Newly diagnosis medical disease & 15 & 0 \\
\hline \multicolumn{3}{|l|}{ Health education } \\
\hline Taught glucose monitoring & 2 & 0 \\
\hline $\mathrm{DM}$ & 8 & 0 \\
\hline Gout & 1 & 0 \\
\hline HT & 3 & 0 \\
\hline Dementia & 3 & 0 \\
\hline Breast cancer/lymphedema & 3 & 0 \\
\hline Healthy lifestyle & 4 & 0 \\
\hline Smoking cessation & 1 & 0 \\
\hline Resources or material provided & 23 & 4 \\
\hline DAMA stayed for treatment & 6 & 0 \\
\hline \multicolumn{3}{|l|}{ Psychological testing } \\
\hline Chinese MCMI & 3 & 3 \\
\hline Projective drawings & 34 & 3 \\
\hline CMMSE & 20 & 0 \\
\hline Montreal & 12 & 0 \\
\hline Bender & 18 & 1 \\
\hline Sentence completion & 13 & 2 \\
\hline IQ test & 0 & 2 \\
\hline Collaboration with social workers & 4 & 0 \\
\hline
\end{tabular}

Note: ADL = activities for daily living; DAMA = discharged against medical advice. other professionals after the initial session of service. Inspection of the frequencies shows that a great deal of time was devoted to mental health needs of patients and staff in an AED setting. This is an aspect of emergency medicine that should not be ignored.

The number of follow-up visit and hours of services visits by service groups was as follows: 13 patients (14.5 hours), 6 staff (6 hours) and 4 relatives (4 hours). Reasons for the consultations with patients included 1) coping with psychosomatic complaints; 2) disease adjustment counseling; 3) diminishing caregivers psychological stress; 4) providing psychoeducation; and/or 5) reducing the need for frequent medical visits. Staff members seeking more than one visit typically had work-related problems such as vicarious trauma resulting from AED situational events (e.g., sudden death of patients, severe injuries, and fatigue) and/or interpersonal work-related disputes.

\subsection{Qualitative Perspectives (Case Vignettes)}

\subsubsection{New Diagnosis of Liver Cancer}

The nursing manager of the emergency medicine ward (EMW) referred somewhat elderly man who had received a diagnosis of a liver carcinoma due to his acute fears and feelings of hopelessness regarding his medical condition. His physiological symptoms included malaise and loss of appetite.

Five sessions were provided with the first centered on suicidal risk assessment followed by an exploration with the patient regarding his understanding regarding the stage

Table 2. Frequency of referrals.

\begin{tabular}{ccc}
\hline CP/counselor & $\begin{array}{c}\text { Patients } \\
(N=281) \\
\text { frequency }\end{array}$ & $\begin{array}{c}\text { Staff } \\
(N=150) \\
\text { frequency }\end{array}$ \\
Geriatric doctor & 28 & 2 \\
Social worker & 9 & 0 \\
Occupational therapist & 7 & 0 \\
Dietitian & 1 & 0 \\
Personal growth course & 3 & 0 \\
Caritas anti-gambling centre & 1 & 3 \\
Cessation smoking centre & 1 & 0 \\
Breast cancer foundation of Hong Kong & 4 & 0 \\
Psychiatric admission/consultation/OPD & 11 & 0 \\
Dementia centre & 8 & 0 \\
Community nurses & 2 & 0 \\
Grief support & 1 & 2 \\
\hline
\end{tabular}

Note: CP = clinical psychologist. 
of his illness along with prognosis and treatment options. Finally, in a collaborative manner, the patient was helped to discover his strengths and resources that could help him attain a meaningful adjustment.

In subsequent sessions a postmodern humanistic approach was used [31] to assist the patient in coming to terms with a life-threatening illness by easing his psychological morbidity and helping him to attain a sense of empowerment. This was followed by solution-focused conversations regarding client-defined outcomes (e.g., improved diet, more positive energy, reduced melancholia). In terms of collaborative work with other professionals, he was referred for psychiatric medication and a dietary assessment.

The outcome of the interventions with the patient was positive and he was able to return to work and engage in family planning for future significant events (e.g., his daughter's upcoming marriage).

\subsubsection{Cognitive Decline}

This case involves a 70-year-old man who was having difficulties in orientation and judgment. For example, although the nursing staff would direct him to his bed several times a day, he often became lost and confused in the EMW. Due to patient management issues, the onduty physician referred the patient to the psychologist for a dementia evaluation. The results of the assessment (Montreal Cognitive Assessment Test, CMMSE) revealed significant cognitive impairments.

In follow-up supportive care sessions, among other issues, it was discovered that the patients had difficulty in understanding and managing his medication regimen. Specifically due to misunderstanding of prescription directives, on a daily basis, he was actually taking only half of his prescribed antihypertensive medications. This had been occurring over the course of nearly one year. To remedy this and other serious problems, psychoeducation techniques were employed (e.g., color drawings of medicines that should be taken on a daily bases, providing family members with information on early dementia care) and this was followed by referral to the geriatric department for additional cognitive assessment and treatment after AED discharge.

\subsubsection{Emergency Family Intervention}

Twenty relatives arrived in the AED to pay their last respect to an elder family member who had committed suicide. The psychologist was then paged to assist staff and the 20 visitors manage a potentially awkward scene in AED. First, the relative were divided into two groups based on the degree of bereavement and potentially soothing others. Once they were in separate rooms, the psychologist employed interventions to diminish the traumatic impact of the self-inflicted death of a beloved fa- mily member (e.g., maintaining a calming and reassuring manner, allowing the expression of grief in a safety zone, assisting in mutual supportive behaviors). Throughout the course of treatment, the psychologist preserved an empathetic attunement in order to prepare the bereaved relatives to pay last respects in an appropriate way given the particular situation in which they found themselves (e.g., police regulations prohibited the touching of the body and limited time in viewing the corpse).

These psychological interventions saved the professional AED medical staff from very difficult and time consuming service management issues. Not surprisingly, there were no time pressures placed on the psychologist as assistance was provided to the family members. It was then suggested that the psychologist provide educative seminars to the medical staff regarding bereavement issues.

\subsubsection{Self-Injurious Youth}

A 13-year-old girl was seen in the AED because of a suicidal gesture of banging her head against a wall resulting in a head injury following an intense argument with her mother. She was referred to the psychologist by the AED attending physician to evaluate her suicidality and mental status. During the assessment period, the patient revealed numerous superficial cutting scars on her wrist.

Initial reserve and appreciation were overcome by a nondirective counseling approach where the youngster was encouraged to complete testing in friendly and nonthreatening environment. It soon became clear that histrionic symptoms were related to a number of family issues including the recent death of her father and unresolved tensions with her mother. Brief therapy regarding emotion regulation was undertaken with the child following by family therapy interventions. The girl was referred to the psychiatrist and the community social worker for followup care.

\section{Discussion}

This research project was designed to explore to the potential utility of health care services provided by a psychologist in an AED of a large and demanding Hong Kong hospital. Utilization findings were encouraging with large numbers of patients and staff seeking and receiving psychological services. It seems likely that when the psychosocial needs of patients are addressed, future attendance at AED and absence from work were substantially reduced in addition to enhance drug adherence [32]. Given that PWH statistics from April 2009 to March 2010 showed that there were 150,530 AED visits, the work of an AED psychologist as described in the present investigation might well results in reductions of repeat hospital attendees which, in turn, would have important financial implications for health care systems serving 
large populations.

It should be remembered that physical conditions are embedded within psychological narratives and these must be understood for medical practice to be effective [33]. In order words, the meaning that a patient attaches to illness and how a malady is integrated into a life chronicle influences many medical treatment outcomes. For example, it has been shown that readmission rates and mortality were significantly diminished along with a number of costly hospital expenses when a biopsychosocial approach are adopted by medical staff [34] and meaning of illness has also been found to be related to compliance issues [35]. Thus, most contemporary physiccians and nurses have little doubt that incorporating psychosocial issues in patient services is a desirable course of action even in busy AED settings. The problem for most medical practitioners providing acute care is time and adequate training. The psychologist could well be a valuable resource providing vital services including 1) suicidal risk screening; 2) cognitive and psychological assessments; 3) assisting clients in possible benefit finding following serious illness; 4) teaching coping skills to patients and staff; 5) bereavement counseling; and 6) offering a channel of communication to significant others at all levels in the complex hospital organization.

According to Wong et al. [36], patient satisfaction levels are influenced by their degree of engagement in decisions about their medical conditions and treatments. Therefore, effective communication between professional staff and consumers should be established and maintained. This can be a daunting task in medical environments where professional staff are dealing with acute emergencies on a continuous bases. For example, AED physicians working in public hospitals typically have to make provisional diagnosis within few minutes as large numbers of patients await critical services. A frenetic pace can take a heavy toll particularly after several hours of continuous work making accommodating dialogues with patients demanding. Having a professional colleague capable of spending extended periods of time with patients engaging in discussions of issues that are important to them has likely benefits for improved quality of care and satisfaction indices.

As this study was conducted in an international setting, cultural issues played an important role in service delivery. Chinese attitudes regarding potential stigma of talking to a mental health professional had to be taken into consideration as were ways physical symptoms are typically expressed, issues of respect and deference to authority figures, and the meaning of illness for patients. It was important to understand folk myths about illnesses, to employ metaphoric language when appropriate, to be sensitive to face issues, and to have knowledge of the social and financial realities faced by ill people in a large

\section{Chinese metropolitan area.}

Chronic occupational stress can easily impair best- practice patient services in high utilization hospitals. Kwok [37], for example, noted three major occupational stressors in AEDs: 1) demanding workload, 2) insufficient resources and 3) conflicts with patients, relatives, and colleagues. Not surprisingly, it has been found that low job satisfaction among AED personnel in Hong Kong is often due to physical and psychological exhaustion [38]. Obviously, the burdens of care among medical caregivers must be addressed by hospital administrators and public health planners in creative ways. Intrastaff mechanisms of peer support and conflict management would go a long way in the cultivation of a positive work environment in pressured AEDs and an on-site psychologist would be useful in this respect.

Informal psychosocial support services for staff might well be a more effective preemptive strategy than traditional employment assistance programs. Early identification and treatment of problematic conditions is as true for psychological problems as it is in medical practice. When a sample of staff members $(N=20)$ were asked about helpful aspects of the psychologist's work, all found services beneficial, particularly availability immediacy. Most valued assistance with personal issues, improved stress management programs (e.g., mindfulness training), and debriefing sessions following difficult situations. Developing nonbureaucratic burnout preventive procedures for at-risk medical practitioners would be a wise course of action and an on-sight psychologist might useful services in this regard.

To fulfill the duties and responsibilities of the psychologist in an AED, the person has to have adequate knowledge of fundamental biological sciences and advanced psychological issues. Training must be sufficient so that collaborative biopsychosocial treatments can be integrated and applied in a short-term emergency treatment setting. In the present circumstance, the psychologist had a nursing background that proved invaluable on many levels (e.g., staff and patient credibility).

It is hoped that this report will stimulate thought regarding new potential roles for psychologists in diverse hospital settings. This, in turn, assists in an even more judicious consideration of body-mind interactions as reflected in a deeper appreciation regarding the potency of "set and setting" variables in medicine [39], biological responses to trauma disclosure [40], and stress issues the progression or alleviation of physical illnesses [41]. Also it provides insights regarding the kinds of psychological services that may be helpful in employee assistance programs for AED (and similar hospital departments) staff.

In terms of limitations, this present study was retrospective and involved only a single hospital site. Addi- 
tionally, data collection was often limited due to confidentially issues. Having more than one psychologist involved would have another improvement. Finally, patients' service satisfaction outcome data were not included in this report.

A randomized control study with pretest-posttest comparisons among various disease and accident groups would be an important next step regarding utility of psychological services in an AED. Inclusion of post-hospitalization variables such as returning to work (RTW) would also strengthen the evaluation of the services of a clinical psychologist in an acute care emergency setting.

\section{Acknowledgements}

Heartfelt appreciation is given to all patients, relatives, and staff for their trust and support which has been enriching in numerous ways.

\section{REFERENCES}

[1] P. Soons and J. Denollet, "Medical Psychology Services in Dutch General Hospitals: State of the Art Developments and Recommendations for the Future," Journal of Clinical Psychology in Medical Settings, Vol. 16, No. 2, 2009, pp. 161-168. doi:10.1007/s10880-009-9158-7

[2] T. W. Smith, P. C. Kendall and F. J. Keefe, "Behavioral Medicine and Clinical Health Psychology: Introduction to the Special Issue, a View from the Decade of Behavior," Journal of Consulting and Clinical Psychology, Vol. 70, No. 3, 2002, pp. 459-462. doi:10.1037/0022-006X.70.3.459

[3] N. Tomes, "The Development of Clinical Psychology, Social Work, and Psychiatric Nursing: 1900-1980s,” In: E. R. Wallace and J. Gach, Eds., History of Psychiatry and Medical Psychology, Springer, New York, pp. 657-682.

[4] A. S. Malik, O. Boyko, N. Atkar and W. F. Young, "A Comparative Study of MR Imaging Profile of Titanium Pedicle Screws,” Acta Radiologica, Vol. 42, No. 3, 2001, pp. 291-293. doi:10.1080/028418501127346846

[5] S. Finger, F. Boller and K. L. Tyler, "History of Clinical Neurology,” Elsevier, Edinburgh, 2010.

[6] T. Stancin, E. C. Perrin and L. Ramirez, "Pediatric Psychology and Primary Care,” In: M. C. Roberts and R. G. Steele, Eds., Handbook of Pediatric Psychology, 4th Edition, Guilford Press, New York, 2009, pp. 630-646.

[7] C. Herrmann-Lingen, "Steps towards Integrated Psychosomatic Medicine-The Example of Psychocardiology," Journal of Psychosomatic Research, Vol. 70, No. 2, 2011, pp. 111-115. doi:10.1016/j.jpsychores.2010.09.019

[8] H. de La Ménardière, M. Reich and L. Vanlemmens, "Reperage, Clinique, et Evaluation de la Depression en Oncologie: Collaboration Psychiatre, Psychologue, et Cancérologue,” Psycho-Oncologie, Vol. 4, No. 4, 2010, pp. 250-255. doi:10.1007/s11839-010-0285-Z

[9] J. A. Morris, "The Rural Psychologist in the Hospital Emergency Room,” In: J. A. Morris Ed., Practicing Psy- chology in Rural Settings: Hospital Privileges and Collaborative Care, American Psychological Association, Washington DC, 1977, pp. 81-96.

[10] G. G. Bodiwala, "Emergency Medicine: A Global Specialty,” Emergency Medicine Australasia, Vol. 19, No. 4, 2007, pp. 387-388.

[11] R. E. Suter, "The Light of Emergency Medicine: Vietnam - The Next Chapter in the Global History of Emergency Medicine?” Academic Emergency Medicine, Vol. 18, No. 4, 2011, pp. e31-e35. doi:10.1111/j.1553-2712.2011.01046.x

[12] International Federation for Emergency Medicine (IFEM), “IFEM Definition of Emergency Medicine,” 2012. http://www.ifem.cc/About_IFEM.aspx

[13] Hong Kong College of Emergency Medicine, "From Casualty to Emergency Medicine-Half a Century of Transformation," Hong Kong Academic of Medicine Press, Hong Kong, 2006.

[14] Hospital Authority of Hong Kong, "Hospital Authority Statistical Report 2010-2011,” 2012. http://www.ha.org.hk/gallery/ha_publications.asp?

[15] R. Burns, A. Cheung, S. Leung and F. Leung, "Management of Accident and Emergency Services: Audit Report,” Hospital Authority, Hong Kong, 2010.

[16] Y. Zhang, H. Su, L. Shang, D. Li, R. Wang, R. Zhang and $\mathrm{Y}$. Xu, "Preferences and Perceived Involvement in Treatment Decision Making among Chinese Patients with Chronic Hepatitis,” An International Journal of the Society for Medical Decision Making, Vol. 31, No. 2, 2011, pp. 245253. doi:10.1177/0272989X10375990

[17] H. Q. Wu, Y. Zang, Y. W. Li, Y. Zhu and F. Shen, “Clinical Characteristics of Patients with Depressive Disorders at Department of Neurology in General Hospitals," Zhonghua Yi Xue Za Zhi, Vol. 19, No. 25, 2010, pp. 1760-1763.

[18] S. B. R. Rosser, "Working as a Psychologist in the Medical Reserve Corps: Providing Emergency Mental Health Relief Services in Hurricanes Katrina and Rita,” Professional Psychology: Research and Practice, Vol. 39, No. 1, 2008, pp. 37-44. doi:10.1037/0735-7028.39.1.37

[19] C. T. Beck, "Secondary Traumatic Stress in Nurses: A Systematic Review," Archives of Psychiatric Nursing, Vol. 25, No. 1, 2011, pp. 1-10. doi:10.1016/j.apnu.2010.05.005

[20] L. M. Culver, B. L. McKinney and L. V. Paradise, “Mental Health Professionals' Experiences of Vicarious Traumatization in Post-Hurricane Katrina New Orleans. Journal of Loss and Trauma, Vol. 16, No. 1, 2011, pp. 33-42. doi:10.1080/15325024.2010.519279

[21] B. Kerasiotis and R. W. Motta, “Assessment of PTSD Symptoms in Emergency Room, Intensive Care Unit, and General Floor Nurses,” International Journal of Emergency Mental Health, Vol. 6, No. 3, 2004, pp. 121-133.

[22] M. Folstein, S. Folstein and P. McHugh, "Mini-Mental State: A Practical Method for Grading the Cognitive State of Patientsfor the Clinician," Journal of Psychiatric Research, Vol. 12, No. 3, 1975, pp. 189-198. doi:10.1016/0022-3956(75)90026-6 
[23] H. F. K. Chiu, H. C. Lee, W. S. Chung and P. K. Kwong, "Reliability and Validity of the Cantonese Version of the Mini-Mental State Examination-A Preliminary Study," Journal of Hong Kong College of Psychiatry, Vol. 4, No. 1, 1994, pp. 25-28.

[24] Z. Nasreddine, N. A. Phillips, V. Bedirian, S. Charbonneau, V. Whitehead, I. Collin and H. Chertkow, "The Montreal Cognitive Assessment, MoCA: A Brief Screening Tool for Mild Cognitive Impairment," Journal of the American Geriatric Society, Vol. 53, No. 4, 2005, pp. 695-699. doi:10.1111/j.1532-5415.2005.53221.X

[25] C. F. Tsai, W. J. Lee, S. J. Wang, B. C Shia, Z. Nasredine and J. L. Fuh, "Psy-chometrics of the Montreal Cognitive Assessment (MoCA) and its Subscales: Validation of the Taiwanese Version of the MoCA and an Item Response Theory Analysis," International Psychogeriatrics, Vol. 24, No. 4, 2012, pp. 651-658. doi:10.1017/S1041610211002298

[26] A. J. Raphael and C. J. Golden, "Rela-tionships of Objectively Scored Bender Variables with MMPI Score in an Outpatient Psychiatric Population," Perceptual and Motor Skills, Vol. 95, No. 3, 2002, pp. 1217-1232. doi:10.2466/pms.2002.95.3f.1217

[27] L. Handler, “The Clinical Use of Figure Drawings,” In: C. S. Newmark, Ed., Major Psychological Assessment Instruments, 2nd Edition, Simon \& Schuster, Needham Heights, 1996, pp. 206-299.

[28] W. M. Patterson, H. H. Dohn, J. Bird and G. A. Patterson, "Evaluation of Suicidal Patients: The SAD PERSONS Scale," Psychometrics: Journal of Consultation Liaison Psychiatry, Vol. 24, No. 4, 1983, pp. 343-349.

[29] S. S. Khan and J. Benger, "SAD PERSONS Scale in Assessing Self-Harm Risk,” Emergency Medicine Journal, Vol. 28, No. 4, 2011, pp. 335-336.

[30] Y. Li, Y. Yang and C. Jiang, “A Study of the Reliability and Vlidity of Millon Clinical Multiaxial Inventory III (MCMI-III)," Chinese Journal of Clinical Psychology, Vol. 18, No. 1, 2010, pp. 11-14.

[31] M. Boulton, M. Boudioni, M. J. Mossman, G. Moynihan, G. Leydon and G. A. Ramirez, "Dividing the Desolation: Clients Views on the Benefits of a Cancer Counseling Service," Psychooncology, Vol. 10, No. 2, 2001, pp. 124136. doi:10.1002/pon.494
[32] J. Pimm, "The Psychological Care of Medical Patients," Clinical Medicine, Vol. 3, No. 2, 2003, pp. 171-172.

[33] J. Skelton, "Narrative Medicine: Stories to Some Purpose,” In: B. D. Kirkcaldy, Ed., The Art and Science of Health Care: Psychology and Human Factors for Practitioners, Hogrefe Publishing, Cambridge, 2011, pp. 313327.

[34] A. P. A. Margalit and A. El-Ad, "Costly Patients with Unexplained Medical Symptoms: A High-Risk Population," Patient Education and Counseling, Vol. 70, No. 2, 2008, pp. 173-178. doi:10.1016/j.pec.2007.09.020

[35] E. D. Peselow, "Adherence with Respect to Medication Management,” Primary Psychiatry, Vol. 14, No. 7, 2007, pp. 83-89.

[36] E. L. Y. Wong, M. C. M. Leung, A. W. L. Cheung, C. H. K. Yam, E. K. Yeoh and S. Griffith, “A Population-Based Survey Using PPE-15: Relationship of Care Aspects to Patient Satisfaction in Hong Kong," International Journal for Quality in Health Care, Vol. 23, No. 4, 2011, pp. 390396. doi:10.1093/intqhc/mzr037

[37] W. O. Kwok, "The Effects of an Intervention Program (MEDI) on Reducing Occupational Stress in Emergency Department Nurses,” PsyD (Clinical), Dissertation, Alliant International University, Los Angeles, 2011.

[38] S. Y. Lee, “Job Burnout among Nurses in Hong Kong: Implications for Human Resource Practices and Interventions," Asia Pacific Journal of Human Resources, Vol. 45, No. 1, 2007, pp. 63-84. doi:10.1177/1038411107073604

[39] B. S. Oken, "Placebo Effects: Clinical Aspects and Neurobiology,” Brain: A Journal of Neurology, Vol. 131, No. 11, 2008, pp. 2812-2823. doi:10.1093/brain/awn116

[40] J. W. Pennebaker, J. K. Kiecolt-Glaser and R. Glaser, "Disclosure of Traumas and Immune Function: Health Implications for Psychotherapy,” In: R. M. Kowalski and M. R. Leary, Eds., The Interface of Social and Clinical Psychology: Key Readings, Psychology Press, New York, 2004, pp. 301-312.

[41] M. H. Antoni, "Stress Management Effects of Psychological, Endocrinological, and Immune Functioning in Men with HIV Infection: Empirical Support for a Sychoneuroimmunological Model,” Stress, Vol. 6, No. 3, 2003, pp. 173-188. doi:10.1080/1025389031000156727 